Journal of Computations \& Modelling, Vol. 11, No. 2, 2021, 1-15

ISSN: $1792-7625$ (print), 1792-8850 (online)

https://doi.org/10.47260/jcomod/1121

Scientific Press International Limited

\title{
Learning Effect on Inventory Model for Deteriorating and Ameliorating Items under Inflation and Partial Backlogging
}

\author{
Biswaranjan Mandal ${ }^{1}$
}

\begin{abstract}
Now-a-days, learning's awareness is increasing in various disciplines because effect of learning has a direct impact on profit or loss, and it is a promotional deemed effective tool for inventory management. The basic concept of the inventory model is that $100 \%$ of the articles in an ordered lot are of good quality but this concept is not practically justifiable for the production process owing to product deterioration and related factors and so deterioration of items cannot be ignored. Again due to lack of considering the influence of demand, the ameliorating items for the amount of inventory is increasing gradually and it is a natural phenomenon observing in much life stock models. In addition, as the deep financial crisis continues to haunt the global economy, the effects of inflation and time value of money cannot be oblivious to an inventory system. Again another important factor is shortages which no retailer would prefer, and in practice are partially backlogged and partially lost. In order to convert the lost sales into sales, the retailer offers such customers an incentive, by charging them the price prevailing at the time of placing an order, instead of the current inflated price. Therefore, bearing in mind these facts, the present paper develops an inventory model for a retailer dealing with deteriorating and ameliorating items with stock dependent demand under the influence of inflation and time-value of money over a fixed planning horizon where holding cost follows the learning curve. Finally, a numerical example is provided to illustrate the proposed model. Comparative study of the optimal solutions with respect to major parameters under different special cases is carried out graphically and some managerial inferences have been presented.
\end{abstract}

Subject classification: AMS Classification No. 90B05.

Keywords: Inventory, Learning effect, Deteriorating, Ameliorating, Inflation, Time-value of money, Shortages and Partial backlogging.

${ }^{1}$ Associate Professor of Mathematics. Acharya Jagadish Chandra Bose College, Kolkata, India.

Article Info: Received: October 16, 2021. Revised: October 28, 2021.

Published online: November 3, 2021. 


\section{Introduction}

In many inventory models, the demand rate of items was often assumed to be either constant or time dependent. However, many researchers have recognized that the demand rate of many retail items is usually influenced by the amount of inventory displayed. For example, Whitin [1] stated, "For retail stores the inventory control problem for style goods is further complicated by the fact that inventory and sales are not independent of one another. An increase in inventories may bring about increased sales of some items." Levin et al. [2] and Sliver and Peterson [3] also observed that sales at the retail level tend to be proportional to the inventory displayed and that large piles of goods displayed in a supermarket will lead customers to buy more. The reason behind this phenomenon is a typical psychology of customers. This may occur due to the fact of feeling of obtaining a wide range for selection when a large amount is stored or displayed or having doubt about the freshness or quality of the product when a small amount is stored. Based on the observed phenomenon, it is cleared that in real life the demand rate of items may be influenced by the stock levels. Many researchers like J. T. Teng et al [4], T. K. Datta et al[5], B. R. Singh [6], R. P. Tripathi et al [7], N. Sharma et al [8] etc have considered stock dependent demand rate in their models.

Again several researchers have addressed the importance of the deterioration phenomenon in their field of applications, as a result, many inventory models with deteriorating items have been developed. But due to lack of considering the influence of demand, the ameliorating items for the amount of inventory is increasing gradually. The fast growing animals like broiler, ducks, pigs etc. in the poultry farm, highbred fishes in berry (pond) which are known as ameliorating items. Amelioration is a natural phenomenon observing in much life stock models. Hwang [9] developed an inventory model for ameliorating items only. Again Hwang [10] added to a stock model for both ameliorating and deteriorating things independently. Mallick et al. [11] has considered a creation inventory model for both ameliorating and deteriorating items. Many researchers like Moon et al[12], Law et al [13], Nodoust [14] are few noteworthy.

In the present field of economy, inflation is a crucial attribute of today's esoteric economy which cannot be shrugged off. The term 'inflation' particularly used in an economic context literally means to blow up or get bigger. However the most common economic meaning of inflation is: reduction in the value of money i.e., monetary depreciation. As a result prices of commodities rise which subsequently curbs the purchasing power. Further from the financial perspective, inventories correspond to substantial investment in capital for any organization; hence it would not be ethical if the effects of inflation and time value of money are not considered while determining the optimal inventory policy. Buzacott [15], Bierman \& Thomas [16], Moon et al [17], Bansal [18], Dr Biswaranjan Mandal [19] are mentioned to few.

Moreover the learning's awareness is increasing in various disciplines because effect of learning has a direct impact on profit or loss, and it is a promotional deemed 
effective tool for inventory management. Few research areas of N. Kumar [20], M. K. Sharma et al [21] etc are important in the field of inventory management.

For these sort of situations, efforts have been made to develop an inventory model in presence of both ameliorating and deteriorating items with stock dependent demand under the influence of inflation and time-value of money over a fixed planning horizon where holding cost follows the learning curve. Shortages are allowed which is partially backlogged. Finally the model is illustrated with the help of a numerical example and the comparative study of the optimal solutions with respect to major parameters under different special cases is carried out graphically and some managerial inferences have been presented.

\section{Assumptions and Nomenclatures}

\subsection{Assumptions}

The present inventory model is developed under the following assumptions:

i. Lead time is zero.

ii. Replenishment rate is infinite but size is finite.

iii. The time horizon is finite.

iv. There is no repair of deteriorated items occurring during the cycle.

v. Amelioration and deterioration occur when the item is effectively in stock.

vi. The demand rate for the product is assumed as stock dependent.

vii. Holding cost follows the learning curve.

viii. Inflation is also considered in this model.

ix. Shortages are allowed and partial backlogged.

\section{$2.2 \quad$ Nomenclatures}

The following nomenclatures are used in the proposed model:

i. $\quad \mathrm{I}(\mathrm{t})$ : On hand inventory at time $\mathrm{t}$.

ii. $\quad \mathrm{D}(\mathrm{t})$ : Demand rate $D(t)=\left|\begin{array}{c}a+b I(t), I(t)>0 \\ a, I(t) \leq 0\end{array}\right|$, where $\mathrm{a}(>0)$ be the initial demand and $\mathrm{b}(0<\mathrm{b}<1)$ be a constant parameter.

iii. $\mathrm{B}(\mathrm{t})$ : Backlogging rate $B(t)=e^{-\delta(T-t)}$, where $\delta(>0)$ is a constant.

iv. Q : On-hand inventory.

v. $\theta$ : The constant deterioration rate where $0 \leq \theta<1$

vi. A : The constant ameliorating rate.

vii. $t_{d}$ : The time length in which the product has no deterioration (fresh product time).

viii. $t_{1}$ : The time length in which the stock is completely diminished.

ix. $\quad \mathrm{T}:$ The fixed length of each production cycle.

x. $\quad \mathrm{i}$ : The inflation rate per unit time. 
xi. r: The discount rate representing the time value of money.

xii. $\quad A_{0}$ : The ordering cost per order during the cycle period.

xiii. $\quad p_{c}$ : The purchasing cost per unit item.

xiv. $\quad h_{0}+\frac{h_{1}}{n^{\alpha_{2}}}$ : The holding cost with learning effect.

xv. $d_{c}$ : The deterioration cost per unit item.

xvi. $\quad a_{c}$ : The cost of amelioration per unit item.

xvii. $\quad c_{s}$ : The shortage cost per unit item.

xviii. $o_{c}$ : The opportunity cost per unit item.

xix. $\quad r_{c}$ : Sales revenue cost per unit time.

$\mathrm{xx}$.

$$
I(t)=\left|\begin{array}{l}
I_{1}(t), 0 \leq t \leq t_{d} \\
I_{2}(t), t_{d} \leq t \leq t_{1} \\
I_{3}(t), t_{1} \leq t \leq T
\end{array}\right|
$$

xxi. TP : Average total profit per unit time.

\section{Mathematical Formulation and Solution}

In this model, we consider an inventory model starting with no shortage. Replenishment occurs at time $\mathrm{t}=0$ and the inventory level attains its maximum. During $\left[0, t_{d}\right]$, the inventory level is depleted only due to stock -dependent demand rate. From $\mathrm{t}=t_{d}$ to $\mathrm{t}=t_{1}$, the stock will be diminished due to the effect of amelioration, deterioration and demand, and ultimately falls to zero at $\mathrm{t}=t_{1}$. The shortages occur during time period $\left[t_{1}, \mathrm{~T}\right]$ which are partially backlogged. The behaviour of the model at any time $t$ can be described by the following differential equations:

$$
\begin{aligned}
& \frac{d I_{1}(t)}{d t}=-\left[a+b I_{1}(t)\right], 0 \leq t \leq t_{d} \\
& \frac{d I_{2}(t)}{d t}+(\theta-A) I_{2}(t)=-\left[a+b I_{2}(t)\right], t_{d} \leq t \leq t_{1} \\
& \text { and } \frac{d I_{3}(t)}{d t}=-a e^{-\delta(T-t)}, t_{1} \leq t \leq T
\end{aligned}
$$


The initial conditions are $I_{1}(0)=Q_{1}, I_{2}\left(t_{1}\right)=0, I_{3}\left(t_{1}\right)=0, I_{3}(T)=-Q_{2}$

and $Q=Q_{1}+Q_{2}$

The solutions of the equations (1), (2) and (3) using (4) are given by the following

$$
I_{1}(t)=Q_{1} e^{-b t}-\frac{a}{b}\left(1-e^{-b t}\right), 0 \leq t \leq t_{d}
$$

$I_{2}(t)=\frac{a}{\theta-A+b}\left\{e^{(\theta-A+b)\left(t_{1}-t\right)}-1\right\}, t_{d} \leq t \leq t_{1}$

and $I_{3}(t)=-a\left\{t-\delta\left(T t-\frac{t^{2}}{2}\right)\right\}+a\left\{t_{1}-\delta\left(T t_{1}-\frac{t_{1}^{2}}{2}\right)\right\}, t_{1} \leq t \leq T$

Since $I_{1}\left(t_{d}\right)=I_{2}\left(t_{d}\right)$, we get the following expression of on-hand inventory from the equations (5) and (6).

$Q_{1}=\frac{a}{b}\left(e^{b t_{d}}-1\right)+\frac{a}{\theta-A+b} e^{b t_{d}}\left\{e^{(\theta-A+b)\left(t_{1}-t_{d}\right)}-1\right\}$,

Again $Q_{2}=-I_{3}(T)$ gives the following expression using

$Q_{2}=a\left(T-\delta \frac{T^{2}}{2}\right)-a\left\{t_{1}-\delta\left(T t_{1}-\frac{t_{1}^{2}}{2}\right)\right\}$

Therefore the total on-hand inventory is given by $Q=Q_{1}+Q_{2}$

or,

$\mathrm{Q}=\frac{a}{b}\left(e^{b t_{d}}-1\right)+\frac{a}{\theta-A+b} e^{b t_{d}}\left\{e^{(\theta-A+b)\left(t_{1}-t_{d}\right)}-1\right\}+$

$a\left(T-\delta \frac{T^{2}}{2}\right)-a\left\{t_{1}-\delta\left(T t_{1}-\frac{t_{1}^{2}}{2}\right)\right\}$

\section{Cost Component}

Based on the above assumptions of the model, the total profit over the period [0, T] consists of the following cost components:

(1) Ordering cost $(\mathrm{OC})$ over the period $[0, \mathrm{~T}]=A_{0}$ (fixed) 
(2) Purchasing cost (PC) over the period $[0, \mathrm{~T}]=p_{c} \mathrm{Q}$ or,

or,

$\mathrm{PC}=p_{c}\left[\frac{a}{b}\left(e^{b t}-1\right)+\frac{a}{\theta-A+b} e^{b t_{d}}\left\{e^{(\theta-A+b)\left(t_{1}-t_{d}\right)}-1\right\}+a\left(T-\delta \frac{T^{2}}{2}\right)-a\left\{t_{1}-\delta\left(T t_{1}-\frac{t_{1}^{2}}{2}\right)\right\}\right]$

(3) Holding cost for carrying inventory (HC) over the period $[0, \mathrm{~T}]$

$\mathrm{HC}=\left(h_{0}+\frac{h_{1}}{n^{\alpha_{2}}}\right)\left[\int_{0}^{t_{d}} I_{1}(t) e^{-R t} d t+\int_{t_{d}}^{t_{1}} I_{2}(t) e^{-R t} d t\right], \mathrm{R}=\mathrm{r}-\mathrm{i}$

Putting the values of $I_{1}(t)$ and $I_{2}(t)$ from (5) and (6), and integrating and then substituting the value of $Q_{1}$ from (8), we get the following:

$\mathrm{HC}=\left(h_{0}+\frac{h_{1}}{n^{\alpha_{2}}}\right)\left[\frac{a}{b+R}\left(e^{b t_{d}}-e^{-R t_{d}}\right)\left[\frac{1}{\theta-A+b}\left\{e^{(\theta-A+b)\left(t_{1}-t_{d}\right)}-1\right\}+\frac{1}{b}\right]-\frac{a}{b R}\left(1-e^{-R t_{d}}\right)\right.$
+
$\left.\frac{a}{\theta-A+b}\left[\frac{1}{\theta-A+b+R}\left\{e^{(\theta-A+b)\left(t_{1}-t_{d}\right)} e^{-R t_{d}}-e^{-R t_{1}}\right\}-\frac{1}{R}\left(e^{-R t_{d}}-e^{-R t_{1}}\right)\right]\right]$

(4) Cost due to deterioration (CD) over the period $[0, T]$

$\mathrm{CD}=d_{c} \theta \int_{t_{d}}^{t_{1}} I_{2}(t) e^{-R t} d t, \mathrm{R}=\mathrm{r}-\mathrm{i}$

Putting the value $I_{2}(t)$ from (6), and then integrating we get the following

$\mathrm{CD}=\frac{d_{c} \theta a}{\theta-A+b}\left[\frac{1}{\theta-A+b+R}\left\{e^{(\theta-A+b)\left(t_{1}-t_{d}\right)} e^{-R t_{d}}-e^{-R t_{1}}\right\}-\frac{1}{R}\left(e^{-R t_{d}}-e^{-R t_{1}}\right)\right]$

(5) The amelioration cost (AMC) over the period $[0, T]$

$\mathrm{AMC}=a_{c} A \int_{t_{d}}^{t_{1}} I_{2}(t) e^{-R t} d t, \mathrm{R}=\mathrm{r}-\mathrm{i}$

Putting the value $I_{2}(t)$ from (6), and then integrating we get the following $\mathrm{AMC}=\frac{a_{c} A a}{\theta-A+b}\left[\frac{1}{\theta-A+b+R}\left\{e^{(\theta-A+b)\left(t_{1}-t_{d}\right)} e^{-R t_{d}}-e^{-R t_{1}}\right\}-\frac{1}{R}\left(e^{-R t_{d}}-e^{-R t_{1}}\right)\right]$

(6) Cost due to shortage (CS) over the period $[0, T]$

$\mathrm{CS}=-c_{s} \int_{t_{1}}^{T} I_{3}(t) e^{-R t} d t, \mathrm{R}=\mathrm{r}-\mathrm{i}$ 
Putting the value $I_{3}(t)$ from (7), and then integrating we get the following

$$
\begin{aligned}
& \mathrm{CS}=-c_{s} \frac{a}{R} e^{-R T}\left(T-\delta T^{2}+\frac{T^{2}}{2}-\frac{\delta T}{R}+\frac{2}{R}+\frac{1}{R^{2}}-t_{1}+\delta T t_{1}-\frac{\delta t_{1}^{2}}{2}\right) \\
& +c_{s} \frac{a}{R} e^{-R t_{1}}\left(\frac{t_{1}^{2}}{2}-\frac{\delta t_{1}^{2}}{2}+\frac{2}{R}+\frac{1}{R^{2}}-\frac{\delta T}{R}\right)
\end{aligned}
$$

(7) Opportunity Cost due to lost sales (OPC) over the period [0,T]

$$
\begin{aligned}
& \mathrm{OPC}=o_{c} \int_{t_{1}}^{T} a\left(1-e^{-\delta(T-t)}\right) e^{-R t} d t \\
& \mathrm{R}=\mathrm{r}-\mathrm{i}=-\frac{o_{c} a}{R}\left(e^{-R T}-e^{-R t_{1}}\right)-\frac{o_{c} a}{\delta-R} e^{-\delta T}\left(e^{(\delta-R) T}-e^{(\delta-R) t_{1}}\right)
\end{aligned}
$$

(8) Sales Revenue Cost (SRC) over the period $[0, \mathrm{~T}]$

$\mathrm{SRC}=r_{c}\left[\int_{0}^{t_{d}}\left\{a+b I_{1}(t)\right\} e^{-R t} d t+\int_{t_{d}}^{t_{1}}\left\{a+b I_{2}(t)\right\} e^{-R t} d t+\int_{t_{1}}^{T} a e^{-\delta(T-t)} e^{-R t} d t\right]$

Putting the values of $I_{1}(t)$ and $I_{2}(t)$ from (5) and (6), and integrating and then substituting the value of $Q_{1}$ from (8), we get the following

$$
\begin{aligned}
& \mathrm{SRC}=\frac{r_{c} a}{R}\left(1-e^{-R t_{1}}\right)+ \\
& \frac{r_{c} a b}{b+R}\left(e^{b t_{d}}-e^{-R t_{d}}\right)\left[\frac{1}{\theta-A+b}\left\{e^{(\theta-A+b)\left(t_{1}-t_{d}\right)}-1\right\}+\frac{1}{b}\right]-\frac{r_{c} a}{R}\left(1-e^{-R t_{d}}\right)+ \\
& \frac{r_{c} a b}{\theta-A+b}\left[\frac{1}{\theta-A+b+R}\left\{e^{(\theta-A+b)\left(t_{1}-t_{d}\right)} e^{-R t_{d}}-e^{-R t_{1}}\right\}-\frac{1}{R}\left(e^{-R t_{d}}-e^{-R t_{1}}\right)\right]+ \\
& \frac{r_{c} a}{\delta-R} e^{-\delta T}\left\{e^{(\delta-R) T}-e^{(\delta-R) t_{1}}\right\}
\end{aligned}
$$


Thus the average total profit per unit time of the system during the cycle $[0, \mathrm{~T}]$ will be

$$
\begin{aligned}
& \left.\mathrm{TP}\left(t_{1}\right)=\frac{1}{T} \text { [ SRC-OC-PC-HC-CD-AMC-CS-OPC }\right] \\
& =\frac{1}{T}\left[\frac{r_{c} a}{R}\left(1-e^{-R t_{1}}\right)+\frac{r_{c} a b}{b+R}\left(e^{b t_{d}}-e^{-R t_{d}}\right)\left[\frac{1}{\theta-A+b}\left\{e^{(\theta-A+b)\left(t_{1}-t_{d}\right)}-1\right\}+\frac{1}{b}\right]-\frac{r_{c} a}{R}\left(1-e^{-R t_{d}}\right)\right. \\
& \frac{r_{c} a b}{\theta-A+b}\left[\frac{1}{\theta-A+b+R}\left\{e^{(\theta-A+b)\left(t_{1}-t_{d}\right)} e^{-R t_{d}}-e^{-R t_{1}}\right\}-\frac{1}{R}\left(e^{-R t_{d}}-e^{-R t_{1}}\right)\right]+\frac{r_{c} a}{\delta-R} e^{-\delta T}\left(e^{(\delta-R) T}-e^{(\delta-R) t_{1}}\right) \\
& \text { - } \quad A_{0}-p_{c}\left[\frac{a}{b}\left(e^{b t}-1\right)+\frac{a}{\theta-A+b} e^{b t_{d}}\left\{e^{(\theta-A+b)\left(t_{1}-t_{d}\right)}-1\right\}+a\left(T-\delta \frac{T^{2}}{2}\right)-a\left\{t_{1}-\delta\left(T t_{1}-\frac{t_{1}^{2}}{2}\right)\right\}\right]- \\
& \text { - } \quad\left(h_{0}+\frac{h_{1}}{n^{\alpha_{2}}}\right)\left[\frac{a}{b+R}\left(e^{b t_{d}}-e^{-R t_{d}}\right)\left[\frac{1}{\theta-A+b}\left\{e^{(\theta-A+b)\left(t_{1}-t_{d}\right)}-1\right\}+\frac{1}{b}\right]-\frac{a}{b R}\left(1-e^{-R t_{d}}\right)\right. \\
& \left.+\frac{a}{\theta-A+b}\left[\frac{1}{\theta-A+b+R}\left\{e^{(\theta-A+b)\left(t_{1}-t_{d}\right)} e^{-R t_{d}}-e^{-R t_{1}}\right\}-\frac{1}{R}\left(e^{-R t_{d}}-e^{-R t_{1}}\right)\right]\right] \\
& \text { - } \frac{d_{c} \theta a}{\theta-A+b}\left[\frac{1}{\theta-A+b+R}\left\{e^{(\theta-A+b)\left(t_{1}-t_{d}\right)} e^{-R t_{d}}-e^{-R t_{1}}\right\}-\frac{1}{R}\left(e^{-R t_{d}}-e^{-R t_{1}}\right)\right] \\
& \text { - } \frac{a_{c} A a}{\theta-A+b}\left[\frac{1}{\theta-A+b+R}\left\{e^{(\theta-A+b)\left(t_{1}-t_{d}\right)} e^{-R t_{d}}-e^{-R t_{1}}\right\}-\frac{1}{R}\left(e^{-R t_{d}}-e^{-R t_{1}}\right)\right] \\
& +c_{s} \frac{a}{R} e^{-R T}\left(T-\delta T^{2}+\frac{T^{2}}{2}-\frac{\delta T}{R}+\frac{2}{R}+\frac{1}{R^{2}}-t_{1}+\delta T t_{1}-\frac{\delta t_{1}^{2}}{2}\right)-c_{s} \frac{a}{R} e^{-R t_{1}}\left(\frac{t_{1}^{2}}{2}-\frac{\delta t_{1}^{2}}{2}+\frac{2}{R}+\frac{1}{R^{2}}-\frac{\delta T}{R}\right) \\
& \left.+\frac{o_{c} a}{R}\left(e^{-R T}-e^{-R t_{1}}\right)+\frac{o_{c} a}{\delta-R} e^{-\delta T}\left(e^{(\delta-R) T}-e^{(\delta-R) t_{1}}\right)\right]
\end{aligned}
$$

To maximize the profit, the necessary condition is $\frac{d T P\left(t_{1}\right)}{d t_{1}}=0$

This gives

$$
\begin{aligned}
& r_{c} a e^{-R t_{1}}\left(1-e^{-\delta\left(T-t_{1}\right)}\right)+\frac{r_{c} a b}{b+R}\left(e^{b t_{d}}-e^{-R t_{d}}\right) e^{(\theta-A+b)\left(t_{1}-t_{d}\right)}+ \\
& \frac{r_{c} a b}{\theta-A+b+R}\left\{e^{(\theta-A+b)\left(t_{1}-t_{d}\right)}-e^{-R t_{1}}\right\} \\
& \text { - } a p_{c}\left\{e^{b t_{d}} e^{(\theta-A+b)\left(t_{1}-t_{d}\right)}+1-\delta\left(T-t_{1}\right)\right\}-\left(h_{0}+\frac{h_{1}}{n^{\alpha_{2}}}\right) \\
& {\left[\frac{a}{b+R}\left(e^{b t_{d}}-e^{-R t_{d}}\right) e^{(\theta-A+b)\left(t_{1}-t_{d}\right)}+\frac{a}{\theta-A+b+R}\left\{e^{(\theta-A+b)\left(t_{1}-t_{d}\right)}-e^{-R t_{1}}\right\}\right]-} \\
& \frac{d_{c} \theta a}{\theta-A+b+R}\left\{e^{(\theta-A+b)\left(t_{1}-t_{d}\right)}-e^{-R t_{1}}\right\}-\frac{a_{c} A a}{\theta-A+b+R}\left\{e^{(\theta-A+b)\left(t_{1}-t_{d}\right)}-e^{-R t_{1}}\right\}- \\
& c_{s} \frac{a}{R}\left\{e^{-R T}\left(1-\delta T+\delta t_{1}\right)+e^{-R t_{1}}\left(\frac{R \delta t_{1}^{2}}{2}-\frac{\delta t_{1}^{2}}{2}+t_{1}-\delta t_{1}-2-\frac{1}{R}+\delta T\right)\right\} \\
& \text { - } o_{c} a e^{-R t_{1}}\left(e^{-\delta\left(T-t_{1}\right)}-1\right)=0
\end{aligned}
$$


For maximum, the sufficient condition $\frac{\mathrm{d}^{2} \mathrm{TP}\left(\mathrm{t}_{1}\right)}{\mathrm{dt}_{1}^{2}}<0$ would be satisfied.

Let $t_{1}=t_{1}^{*}$ be the optimum value of $t^{t_{1}}$.

The optimal values $Q^{*}$ of $\mathrm{Q}$ and $T P^{*}$ of TP are obtained from the expressions (10) and (19) by putting the value $t_{1}=t_{1}{ }^{*}$.

\section{Some Special Cases}

a) Absence of deterioration

If the deterioration of items is switched off i.e. $\theta=0$, then the expressions (10) and (19) of on-hand inventory $(\mathrm{Q})$ and average total profit per unit time $\left(\operatorname{TP}\left(t_{1}\right)\right)$ during the period $[0, \mathrm{~T}]$ become

$$
\begin{aligned}
& \mathrm{Q}=\frac{a}{b}\left(e^{b t_{d}}-1\right)+\frac{a}{b-A} e^{b t_{d}}\left\{e^{(b-A)\left(t_{1}-t_{d}\right)}-1\right\}+ \\
& a\left(T-\delta \frac{T^{2}}{2}\right)-a\left\{t_{1}-\delta\left(T t_{1}-\frac{t_{1}^{2}}{2}\right)\right\}
\end{aligned}
$$

And

$$
\begin{aligned}
& \operatorname{TP}\left(t_{1}\right)= \frac{1}{T}\left[\frac{r_{c} a}{R}\left(1-e^{-R t_{1}}\right)+\frac{r_{c} a b}{b+R}\left(e^{b t_{d}}-e^{-R t_{d}}\right)\left[\frac{1}{b-A}\left\{e^{(b-A)\left(t_{1}-t_{d}\right)}-1\right\}+\frac{1}{b}\right]-\frac{r_{c} a}{R}\left(1-e^{-R t_{d}}\right)\right. \\
& \frac{r_{c} a b}{b-A}[\left.\frac{1}{b-A+R}\left\{e^{(b-A)\left(t_{1}-t_{d}\right)} e^{-R t_{d}}-e^{-R t_{1}}\right\}-\frac{1}{R}\left(e^{-R t_{d}}-e^{-R t_{1}}\right)\right]+\frac{r_{c} a}{\delta-R} e^{-\delta T}\left(e^{(\delta-R) T}-e^{(\delta-R) t_{1}}\right) \\
&-\left.A_{0}-p_{c}\left[\frac{a}{b}\left(e^{b t}-1\right)+\frac{a}{b-A} e^{b t_{d}}\left\{e^{(b-A)\left(t_{1}-t_{d}\right)}-1\right\}+a\left\{T-\delta \frac{T^{2}}{2}\right)\right\}-a\left\{t_{1}-\delta\left(T t_{1}-\frac{t_{1}^{2}}{2}\right)\right\}\right]- \\
&-\quad\left(h_{0}+\frac{h_{1}}{n^{\alpha_{2}}}\right)\left[\frac{a}{b+R}\left(e^{b t_{d}}-e^{-R t_{d}}\right)\left[\frac{1}{b-A}\left\{e^{(b-A)\left(t_{1}-t_{d}\right)}-1\right\}+\frac{1}{b}\right]-\frac{a}{b R}\left(1-e^{-R t_{d}}\right)\right. \\
&+\left.\frac{a}{b-A}\left[\frac{1}{b-A+R}\left\{e^{(b-A)\left(t_{1}-t_{d}\right)} e^{-R t_{d}}-e^{-R t_{1}}\right\}-\frac{1}{R}\left(e^{-R t_{d}}-e^{-R t_{1}}\right)\right]\right] \\
&-\quad \frac{a_{c} A a}{b-A}\left[\frac{1}{b-A+R}\left\{e^{(b-A)\left(t_{1}-t_{d}\right)} e^{-R t_{d}}-e^{-R t_{1}}\right\}-\frac{1}{R}\left(e^{-R t_{d}}-e^{-R t_{1}}\right)\right] \\
&+c_{s} \frac{a}{R} e^{-R T}\left(T-\delta T^{2}+\frac{T^{2}}{2}-\frac{\delta T}{R}+\frac{2}{R}+\frac{1}{R^{2}}-t_{1}+\delta T t_{1}-\frac{\delta t_{1}^{2}}{2}\right)-c_{s} \frac{a}{R} e^{-R t_{1}}\left(\frac{t_{1}^{2}}{2}-\frac{\delta t_{1}^{2}}{2}+\frac{2}{R}+\frac{1}{R^{2}}-\frac{\delta T}{R}\right) \\
&\left.\quad+\frac{o_{c} a}{R}\left(e^{-R T}-e^{-R t_{1}}\right)+\frac{o_{c} a}{\delta-R} e^{-\delta T}\left(e^{(\delta-R) T}-e^{(\delta-R) t_{1}}\right)\right]
\end{aligned}
$$


The equation (20) becomes

$$
\begin{aligned}
& r_{c} a e^{-R t_{1}}\left(1-e^{-\delta\left(T-t_{1}\right)}\right)+\frac{r_{c} a b}{b+R}\left(e^{b t_{d}}-e^{-R t_{d}}\right) e^{(b-A)\left(t_{1}-t_{d}\right)}+\frac{r_{c} a b}{b-A+R}\left\{e^{(b-A)\left(t_{1}-t_{d}\right)}-e^{-R t_{1}}\right\} \\
& \quad-\quad a p_{c}\left\{e^{b t_{d}} e^{(b-A)\left(t_{1}-t_{d}\right)}+1-\delta\left(T-t_{1}\right)\right\}-\left(h_{0}+\frac{h_{1}}{n^{\alpha_{2}}}\right)\left[\frac{a}{b+R}\left(e^{b t_{d}}-e^{-R t_{d}}\right) e^{(b-A)\left(t_{1}-t_{d}\right)}\right. \\
& \left.+\frac{a}{b-A+R}\left\{e^{(b-A)\left(t_{1}-t_{d}\right)}-e^{-R t_{1}}\right\}\right]-\frac{a_{c} A a}{b-A+R}\left\{e^{(b-A)\left(t_{1}-t_{d}\right)}-e^{-R t_{1}}\right\}- \\
& \quad c_{s} \frac{a}{R}\left\{e^{-R T}\left(1-\delta T+\delta t_{1}\right)+e^{-R t_{1}}\left(\frac{R \delta t_{1}^{2}}{2}-\frac{\delta t_{1}^{2}}{2}+t_{1}-\delta t_{1}-2-\frac{1}{R}+\delta T\right)\right\}- \\
& o_{c} a e^{-R t_{1}}\left(e^{-\delta\left(T-t_{1}\right)}-1\right)=0
\end{aligned}
$$

This gives the optimum value of $t_{1}$.

b) Absence of amelioration

If the amelioration of items is switched off i.e. $A=0$, then the expressions (10) and (19) of on-hand inventory $(\mathrm{Q})$ and average total profit per unit time $\left(\operatorname{TP}\left(t_{1}\right)\right)$ during the period $[0, \mathrm{~T}]$ become

$$
\begin{aligned}
& \mathrm{Q}=\frac{a}{b}\left(e^{b t_{d}}-1\right)+\frac{a}{\theta+b} e^{b t_{d}}\left\{e^{(\theta+b)\left(t_{1}-t_{d}\right)}-1\right\}+ \\
& a\left(T-\delta \frac{T^{2}}{2}\right)-a\left\{t_{1}-\delta\left(T t_{1}-\frac{t_{1}^{2}}{2}\right)\right\}
\end{aligned}
$$

and

$$
\begin{aligned}
& \mathrm{Q}=\frac{a}{b}\left(e^{b t_{d}}-1\right)+\frac{a}{\theta+b} e^{b t_{d}}\left\{e^{(\theta+b)\left(t_{1}-t_{d}\right)}-1\right\}+a\left(T-\delta \frac{T^{2}}{2}\right)-a\left\{t_{1}-\delta\left(T t_{1}-\frac{t_{1}^{2}}{2}\right)\right\} \\
& \text { And TP }\left(t_{1}\right)=\frac{1}{T}\left[\frac{r_{c} a}{R}\left(1-e^{-R t_{1}}\right)+\frac{r_{c} a b}{b+R}\left(e^{b t_{d}}-e^{-R t_{d}}\right)\left[\frac{1}{\theta+b}\left\{e^{(\theta+b)\left(t_{1}-t_{d}\right)}-1\right\}+\frac{1}{b}\right]-\frac{r_{c} a}{R}\left(1-e^{-R t_{d}}\right)\right. \\
& \frac{r_{c} a b}{\theta+b}\left[\frac{1}{\theta+b+R}\left\{e^{(\theta+b)\left(t_{1}-t_{d}\right)} e^{-R t_{d}}-e^{-R t_{1}}\right\}-\frac{1}{R}\left(e^{-R t_{d}}-e^{-R t_{1}}\right)\right]+\frac{r_{c} a}{\delta-R} e^{-\delta T}\left(e^{(\delta-R) T}-e^{(\delta-R) t_{1}}\right) \\
& \text { - } \left.\quad A_{0}-p_{c}\left[\frac{a}{b}\left(e^{b t}-1\right)+\frac{a}{\theta+b} e^{b t_{d}}\left\{e^{(\theta+b)\left(t_{1}-t_{d}\right)}-1\right\}+a\left\{T-\delta \frac{T^{2}}{2}\right)\right\}-a\left\{t_{1}-\delta\left(T t_{1}-\frac{t_{1}^{2}}{2}\right)\right\}\right]- \\
& -\left(h_{0}+\frac{h_{1}}{n^{\alpha_{2}}}\right)\left[\frac{a}{b+R}\left(e^{b t_{d}}-e^{-R t_{d}}\right)\left[\frac{1}{\theta+b}\left\{e^{(\theta+b)\left(t_{1}-t_{d}\right)}-1\right\}+\frac{1}{b}\right]-\frac{a}{b R}\left(1-e^{-R t_{d}}\right)\right. \\
& \left.+\frac{a}{\theta+b}\left[\frac{1}{\theta+b+R}\left\{e^{(\theta+b)\left(t_{1}-t_{d}\right)} e^{-R t_{d}}-e^{-R t_{1}}\right\}-\frac{1}{R}\left(e^{-R t_{d}}-e^{-R t_{t_{1}}}\right)\right]\right]-\frac{d_{c} \theta a}{\theta+b}\left[\frac{1}{\theta+b+R}\left\{e^{(\theta+b)\left(t_{1}-t_{d}\right)} e^{-R_{t_{d}}}-e^{-R_{t_{t}}}\right\}-\frac{1}{R}\left(e^{-R_{t_{d}}}-e^{-R R_{1}}\right)\right] \\
& +c_{s} \frac{a}{R} e^{-R T}\left(T-\delta T^{2}+\frac{T^{2}}{2}-\frac{\delta T}{R}+\frac{2}{R}+\frac{1}{R^{2}}-t_{1}+\delta T t_{1}-\frac{\delta t_{1}^{2}}{2}\right)-c_{s} \frac{a}{R} e^{-R t_{1}}\left(\frac{t_{1}^{2}}{2}-\frac{\delta t_{1}^{2}}{2}+\frac{2}{R}+\frac{1}{R^{2}}-\frac{\delta T}{R}\right) \\
& \left.+\frac{o_{c} a}{R}\left(e^{-R T}-e^{-R t_{1}}\right)+\frac{o_{c} a}{\delta-R} e^{-\delta T}\left(e^{(\delta-R) T}-e^{(\delta-R) t_{1}}\right)\right]
\end{aligned}
$$


The equation (20) becomes

$$
\begin{aligned}
& r_{c} a e^{-R t_{1}}\left(1-e^{-\delta\left(T-t_{1}\right)}\right)+\frac{r_{c} a b}{b+R}\left(e^{b t_{d}}-e^{-R t_{d}}\right) e^{(\theta+b)\left(t_{1}-t_{d}\right)}+\frac{r_{c} a b}{\theta+b+R}\left\{e^{(\theta+b)\left(t_{1}-t_{d}\right)}-e^{-R t_{1}}\right\} \\
& -a_{c}\left\{e^{b t_{d}} e^{(\theta+b)\left(t_{1}-t_{d}\right)}+1-\delta\left(T-t_{1}\right)\right\}-\left(h_{0}+\frac{h_{1}}{n^{\alpha_{2}}}\right)\left[\frac{a}{b+R}\left(e^{b t_{d}}-e^{-R t_{d}}\right) e^{(\theta+b)\left(t_{1}-t_{d}\right)}+\right. \\
& \left.\frac{a}{\theta+b+R}\left\{e^{(\theta+b)\left(t_{1}-t_{d}\right)}-e^{-R t_{1}}\right\}\right]-\frac{d_{c} \theta a}{\theta+b+R}\left\{e^{(\theta+b)\left(t_{1}-t_{d}\right)}-e^{-R t_{1}}\right\}- \\
& c_{s} \frac{a}{R}\left\{e^{-R T}\left(1-\delta T+\delta t_{1}\right)+e^{-R t_{1}}\left(\frac{R \delta t_{1}^{2}}{2}-\frac{\delta t_{1}^{2}}{2}+t_{1}-\delta t_{1}-2-\frac{1}{R}+\delta T\right)\right\}-o_{c} a e^{-R t_{1}}\left(e^{-\delta\left(T-t_{1}\right)}-1\right)=0
\end{aligned}
$$

This gives the optimum value of $t_{1}$.

c) Constant demand rate

If the demand rate is constant in nature i.e. $b=0$, then the expressions (10) and (19) of on-hand inventory $(\mathrm{Q})$ and average total profit per unit time $\left(\mathrm{TP}\left(t_{1}\right)\right)$ during the period $[0, \mathrm{~T}]$ become

$$
\begin{aligned}
& \mathrm{Q}=a t_{d}+\frac{a}{\theta-A}\left\{e^{(\theta-A)\left(t_{1}-t_{d}\right)}-1\right\}+a\left(T-\delta \frac{T^{2}}{2}\right)-a\left\{t_{1}-\delta\left(T t_{1}-\frac{t_{1}^{2}}{2}\right)\right\} \\
& \text { and TP }\left(t_{1}\right)=\frac{1}{T}\left[\frac{r_{c} a}{R}\left(1-e^{-R t_{1}}\right)+\frac{r_{c} a}{\delta-R} e^{-\delta T}\left(e^{(\delta-R) T}-e^{(\delta-R) t_{1}}\right)-A_{0}\right. \\
& -p_{c} a\left[t_{d}+\frac{1}{\theta-A}\left\{e^{(\theta-A)\left(t_{1}-t_{d}\right)}-1\right\}+\left(T-\delta \frac{T^{2}}{2}\right)-\left\{t_{1}-\delta\left(T t_{1}-\frac{t_{1}^{2}}{2}\right)\right\}\right] \\
& -\left(h_{0}+\frac{h_{1}}{n^{\alpha_{2}}}\right)\left[\frac{a}{R}\left(1-e^{-R t_{d}}\right)\left[\frac{1}{\theta-A}\left\{e^{(\theta-A)\left(t_{1}-t_{d}\right)}-1\right\}+t_{d}-1-\frac{1}{R}\right]\right. \\
& \left.+\frac{a}{\theta-A}\left[\frac{1}{\theta-A+R}\left\{e^{(\theta-A)\left(t_{1}-t_{d}\right)} e^{-R t_{d}}-e^{-R t_{1}}\right\}-\frac{1}{R}\left(e^{-R t_{d}}-e^{-R t_{1}}\right)\right]\right] \\
& -\frac{d_{c} \theta a}{\theta-A}\left[\frac{1}{\theta-A+R}\left\{e^{(\theta-A)\left(t_{1}-t_{d}\right)} e^{-R t_{d}}-e^{-R t_{1}}\right\}-\frac{1}{R}\left(e^{-R t_{d}}-e^{-R t_{1}}\right)\right] \\
& -\frac{a_{c} A a}{\theta-A}\left[\frac{1}{\theta-A+R}\left\{e^{(\theta-A)\left(t_{1}-t_{d}\right)} e^{-R t_{d}}-e^{-R t_{1}}\right\}-\frac{1}{R}\left(e^{-R t_{d}}-e^{-R t_{1}}\right)\right] \\
& +c_{s} \frac{a}{R} e^{-R T}\left(T-\delta T^{2}+\frac{T^{2}}{2}-\frac{\delta T}{R}+\frac{2}{R}+\frac{1}{R^{2}}-t_{1}+\delta T t_{1}-\frac{\delta t_{1}^{2}}{2}\right) \\
& -c_{s} \frac{a}{R} e^{-R t_{1}}\left(\frac{t_{1}^{2}}{2}-\frac{\delta t_{1}^{2}}{2}+\frac{2}{R}+\frac{1}{R^{2}}-\frac{\delta T}{R}\right) \\
& \left.+\frac{o_{c} a}{R}\left(e^{-R T}-e^{-R t_{1}}\right)+\frac{o_{c} a}{\delta-R} e^{-\delta T}\left(e^{(\delta-R) T}-e^{(\delta-R) t_{1}}\right)\right]
\end{aligned}
$$


The equation (20) becomes

$$
\begin{aligned}
& r_{c} a e^{-R t_{1}}\left(1-e^{-\delta\left(T-t_{1}\right)}\right)-a p_{c}\left\{e^{(\theta-A)\left(t_{1}-t_{d}\right)}+1-\delta\left(T-t_{1}\right)\right\}-\left(h_{0}+\frac{h_{1}}{n^{\alpha_{2}}}\right) \\
& {\left[\frac{a}{R}\left(1-e^{-R t_{d}}\right) e^{(\theta-A)\left(t_{1}-t_{d}\right)}+\frac{a}{\theta-A+R}\left\{e^{(\theta-A)\left(t_{1}-t_{d}\right)}-e^{-R t_{1}}\right\}\right]} \\
& -\frac{d_{c} \theta a}{\theta-A+R}\left\{e^{(\theta-A)\left(t_{1}-t_{d}\right)}-e^{-R t_{1}}\right\}-\frac{a_{c} A a}{\theta-A+R}\left\{e^{(\theta-A)\left(t_{1}-t_{d}\right)}-e^{-R t_{1}}\right\} \\
& -c_{s} \frac{a}{R}\left\{e^{-R T}\left(1-\delta T+\delta t_{1}\right)+e^{-R t_{1}}\left(\frac{R \delta t_{1}^{2}}{2}-\frac{\delta t_{1}^{2}}{2}+t_{1}-\delta t_{1}-2-\frac{1}{R}+\delta T\right)\right\} \\
& -o_{c} a e^{-R t_{1}}\left(e^{-\delta\left(T-t_{1}\right)}-1\right)=0
\end{aligned}
$$

This gives the optimum value of $t_{1}$.

\section{Numerical Analysis}

To exemplify the above model numerically, let the values of parameters be as follows:

$\mathrm{a}=200 ; \mathrm{b}=0.5 ; \theta=0.02 ; \mathrm{A}=0.01 ; \delta=10 ; A_{0}=\$ 100$ per order; $p_{c}=\$ 5$ per unit, $h_{0}=\$ 2.5$ per unit; $h_{1}=\$ 2.5$ per unit; $\mathrm{n}=3 ; \quad \alpha_{2}=0.1 ; d_{c}=\$ 9$ per unit; $a_{c}=\$ 6$ per unit; $c_{s}=\$ 10$ per unit; $o_{c}=\$ 12$ per unit; $\mathrm{i}=0.08 ; \mathrm{r}=2 ; r_{c}=\$ 12$ per unit; $t_{d}=0.2$ year and $\mathrm{T}=1$ year

Solving the equation (20) with the help of computer using the above values of parameters, we find the following optimum outputs

$$
t_{1}^{*}=0.74 \text { year; } Q^{*}=160.51 \text { units and } T P^{*}=\text { Rs. } 265.85
$$

It is checked that this solution satisfies the sufficient condition for optimality.

On the basis of the above parameters, the following solutions are also made.

\begin{tabular}{|l|c|c|c|}
\hline \multicolumn{1}{|c|}{ Special cases } & $t_{1}^{*}$ & $Q^{*}($ units $)$ & $T P^{*}(\mathbf{\$})$ \\
\hline Absence of deterioration & 0.80 & 165.79 & 225.73 \\
\hline Absence of amelioration & 0.73 & 160.15 & 268.66 \\
\hline The constant demand rate & 0.77 & 147.41 & 99.30 \\
\hline
\end{tabular}




\section{Comparative study of the optimal solutions towards different special cases in the inventory model}

The comparative study is also furnished graphically to illustrate the special cases of the inventory model by varying nature of inventory models.

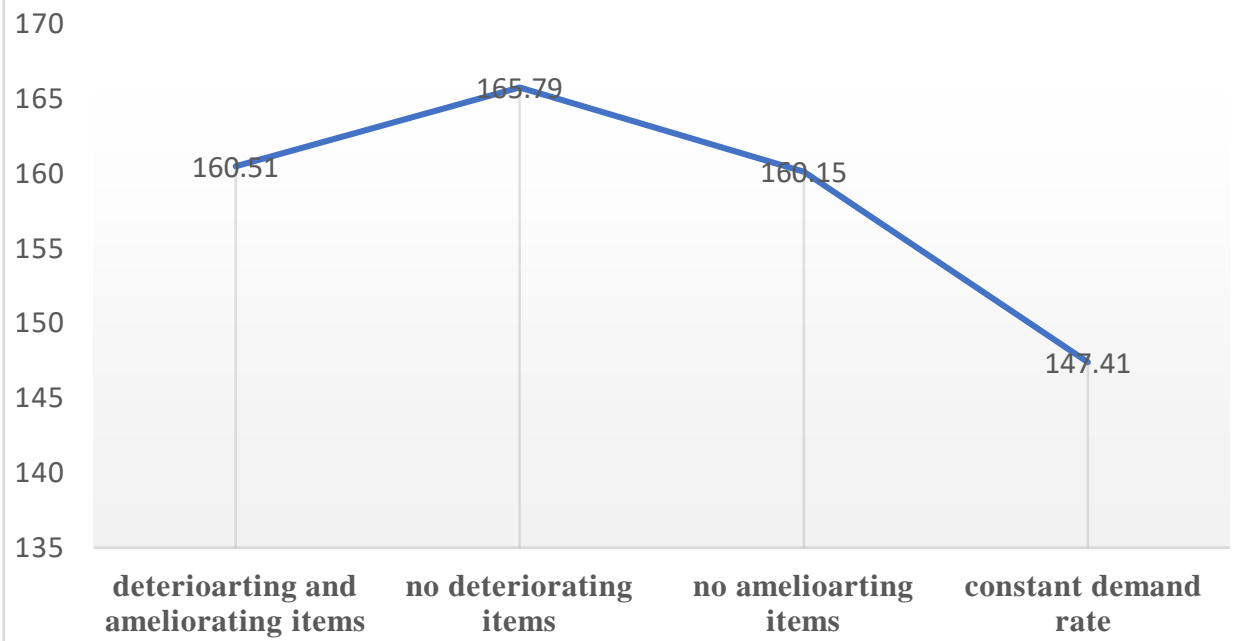

Figure 1: Inventory items vs Optimal On-hand Inventory

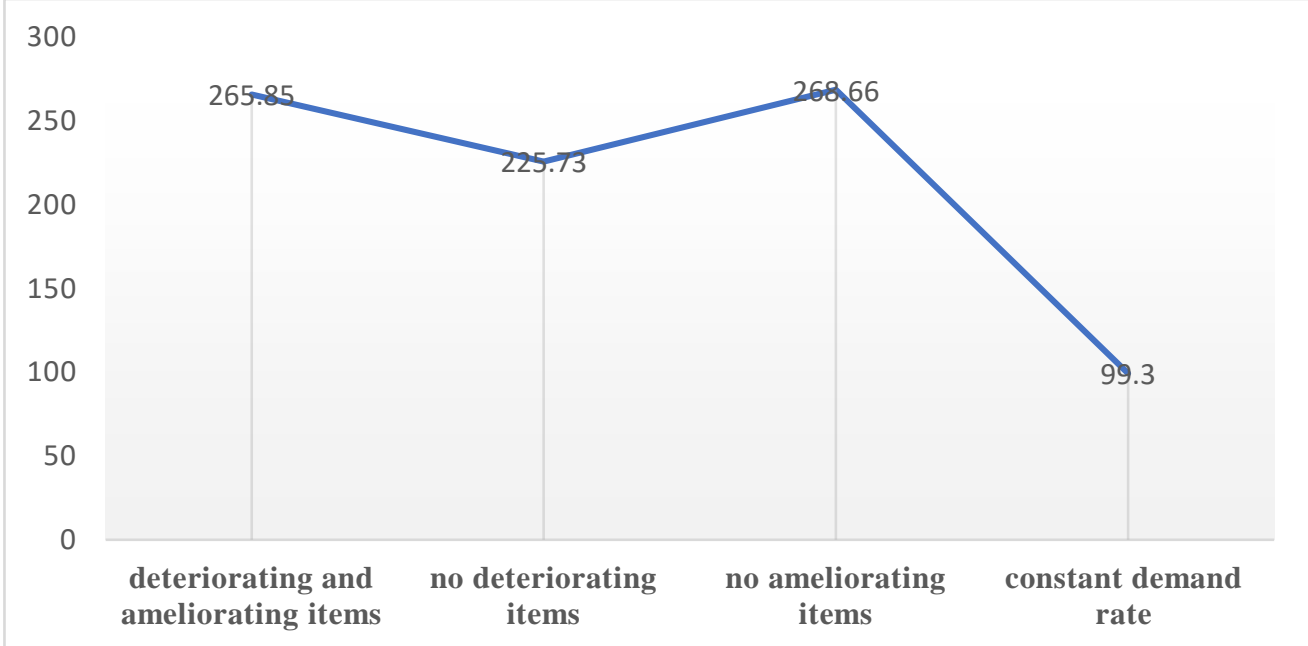

Figure 2: Inventory items vs Optimal Inventory Total Profit 


\section{Observations}

The present model deals with the learning effect in presence of both ameliorating and deteriorating items under stock dependent demand assuming the influence of inflation and time-value of money over a fixed planning horizon. Shortages are allowed which are partially backlogged. The model is also developed analytically and graphically considering some special cases like model with deteriorating and ameliorating, no deteriorating, no ameliorating and constant rate of demand. Analyzing Fig. 1 and Fig. 2, it is observed that optimality of inventory total profit and on-hand inventory level are changing very less sensitively for all kinds of inventory goods except model where the demand rate is constant in nature. Moreover it is also observed that the optimal inventory total profit is significantly very high for the inventory model where the demand rate is trended as constant in nature. So demand parameter plays an important role for estimation of the optimality of inventory profit.

\section{References}

[1] Whitin, T. M. (1957). The Theory of Inventory Management, Princeton University Press, Princeton, NJ, USA.

[2] Levin, R. I., McLaughlin, C. P., Lamone, R. P. and Kottas, J. F. (1972). Production/Operations Management: Contemporary Policy forManaging Operating Systems, McGraw-Hill, New York, NY, USA.

[3] Silver, E. A. and Peterson, R. (1985). "Decision Systems for Inventory Management and Production Planning, Wiley, New York, NY, USA.

[4] Teng, J. T. and Chang, C. T. (1995). "EPQ model for deteriorating items with price and stock-dependent demand" Computers and Operations Research, 31, pp. 297-308.

[5] Datta, T. K. and Pal, A. K. (1998). "Demand proportion by up-gradation under stock-dependent demand situation- a model”, Int. J Prod. Eco., 55, pp. 31-38.

[6] Singh, B. R., Singh, C. and Singh, T. J. (2007). "Optimal policy for decaying items with stock-dependent demand under inflation in a supply chain", Int. review of Pure and Applied Mathematics, 3(2), pp. 189-197.

[7] Tripathi, R. P., Singh, D. and Surbhi, A. (2017). "Inventory models for stockdependent demand and time varying holding cost under different trade credits", Yugoslav Journal of Operations Research.

DOI: https://doi.org/10.2298/YJOR160317018T, pp. 1-13.

[8] Sharma, N. and Vrat, P. (2018). "Inventory models with stock-dependent demand: a comprehensive review and its linkage with waste management", International Journal of Inventory Research, 5(2), pp.112 - 152.

[9] Hwang, H. S. (1997). "A study of an inventory model for items with Weibull ameliorating", Computers and Industrial Engineering, 33, pp.701-704.

[10] Hwang, H. S. (2004). "A stochastic set-covering location model for both ameliorating and deteriorating items", Computers and Industrial Engineering, 46, pp.313-319. 
[11] Mallick, M., Mishra, S., Mishra, U. K. and Paikray, S. K. (2018). "Optimal inventory control for ameliorating, deteriorating items under time varying demand condition", Journal of Social Science Research, 3(1), pp.166-173.

[12] Moon, I., Giri, B.C. and Ko, B. (2005). " Economic order quantity models for ameliorating/deteriorating items under inflation and time discounting", European Journal of Operational Research, 162(3), pp. 773-785.

[13] Law, S. T. and Wee, H.M. (2006). "An integrated production-inventory model for ameliorating and deteriorating items taking account of time discounting", Mathematical and Computer Modelling, vol. 43, no. 5-6, pp. 673-685.

[14] Nodoust, S., Mirzazadeh, A. and Weber, G. (2017). "An evidential reasoning approach for production modeling with deteriorating and ameliorating items", Operational Research.

[15] Buzacott, J. A. (1975). "Economic order quantities with inflation", Operational Research Quarterly, 26(3), pp. 553-558.

[16] Bierman, H. and Thomas, J. (1977). "Inventory decisions under inflationary conditions", Decision Sciences, 8(1), pp. 151-155.

[17] Moon, I., Giri, B. C. and Ko, B. (2005). "Economic order quantity models for ameliorating/deteriorating items under inflation and time discounting", European Journal of Operational Research, 162(3), pp. 773-785.

[18] Bansal, K. K. (2013). 'Inventory Model for Deteriorating Items with the Effect of inflation", International Journal of Application or Innovation in Engineering \& Management (IJAIEM), 2(5), May, pp. 143-150.

[19] Mandal, B. (2020). "An Inflationary Trended Inventory Model for Deteriorating and Ameliorating Items under Exponentially Increasing Demand and Partial Backlogging", International Journal of Application or Innovation in Engineering \& Management (IJAIEM), Web Site: www.ijaiem.org Email: editor@ijaiem.org, 9(12), pp. 1-10.

[20] Kumar, N. (2013). "Learning effect on an inventory model with two-level storage and partial backlogging under inflation", Int. J. Services and Operations Management, 16(1), pp. 105-122

[21] Sharma, M. K. and Bansal, K. K. (2017). "Inventory Model for NonInstantaneous Decaying Items with Learning Effect under Partial Backlogging and Inflation", Global Journal of Pure and Applied Mathematics. ISSN 09731768 13(6), pp. 1999-2008. 